Computers \& Chemical Engineering , Volume 60, 10 J anuary 2014, Pages 297-306

\title{
Adaptive Mesh Refinement of Gas-Liquid Flow on an Inclined Plane
}

\author{
J.J. Cooke ${ }^{\mathrm{a}}$, L.M. Armstrong ${ }^{\mathrm{a}}$, K.H. Luo ${ }^{\mathrm{a}}$, S. Gu ${ }^{\mathrm{b}, *}$ \\ ${ }^{a}$ Energy Technology Research Group, School of Engineering Sciences, University of \\ Southampton, Southampton SO17 1BJ, United Kingdom \\ ${ }^{b}$ School of Engineering, Cranfield University, Cranfield, Bedfordshire MK43 0AL, United \\ Kingdom
}

\begin{abstract}
Carbon Capture \& Storage (CCS) is one of the various methods that can be used to reduce the carbon footprint of the energy sector. The efficiency with which $\mathrm{CO} 2$ is absorbed from flue gas using packed columns is highly dependent on the structure of the liquid films that form on the packing materials. This work examines the hydrodynamics of these liquid films using the CFD solver, OpenFOAM to solve two-phase, isothermal, non-reacting flow using the volume-of-fluid (VOF) method. Local adaptive mesh refinement (AMR) is used to ensure improved resolution of the geometrical grids at the gas-liquid interface. Comparisons are made between the solutions obtained using AMR and those obtained using highly refined static meshes. It was observed that local AMR produced results with much better correlation to experimental data.
\end{abstract}

Keywords:

CCS, AMR, liquid film flow, packed columns, wetted area

\section{Introduction}

The variety of approaches used to separate $\mathrm{CO} 2$ during energy production from hydrocarbon fuels and its subsequent storage away from the atmosphere are known as Carbon Capture \& Storage (CCS). The post-combustion capture of $\mathrm{CO} 2$ can be achieved by reacting flue gas with an amine solution, pre-

\footnotetext{
*Corresponding author

Email address: s.gu@cranfield.ac.uk (Tel:+441234 755277) (S. Gu)

Preprint submitted to Journal of Computers and Chemical Engineering August 13, 2013

Published by Elsevier, Author Accepted M anuscript issued with:

Creative Commons Attribution Non-Commercial License No Derivatives (CC:BY:NC:ND 3.0).
} 
dominantly monoethanolamine (MEA). The reaction of CO2 with aqueous MEA is a reversible reaction, allowing the CO2 to be recovered and transported to storage facilities. CO2 is removed from flue gases in an absorber column, where cooled MEA flows counter-current to the gas flow. The amine solution is subsequently heated in a stripper using a counter-current flow of steam. This process shifts the chemical equilibrium of the system, thereby releasing the CO2 from the amine solution (Freguia \& Rochelle, 2003). This regenerates the amine solution which is cooled prior to being re-introduced into the absorber column.

The rate of absorption of $\mathrm{CO} 2$ is highly dependent on the structure of the liquid film within the packed columns. In particular, a larger gas-liquid interfacial surface area will result in increased absorption of CO2 across the interface. The packing material within packed columns are designed to provide large gas-liquid interfacial areas, therefore enhancing heat and mass transfer. Structured packings are considered optimal for CCS because they have high geometrical areas per unit volume and large void fractions (Raynal et al., 2004). Therefore, they provide the large surface areas required, whilst maintaining low pressure drop (Alix \& Raynal, 2008).

The large range of spatial scales within packed column reactors introduces major difficulties when modelling the process using computational fluid dynamics (CFD). In particular, due to computing power limitations, the effect of small-scale packing surface texture on the hydrodynamics of the system cannot be resolved simultaneously with the hydrodynamics of the whole column. These difficulties can be overcome by making simplifications to the model to determine specific characteristics of the flow. For example, analysis of the liquid films throughout the column can be performed ignoring reaction kinetics, under the assumption that reactions within the system do not affect the structure of the liquid films.

Alternatively, the domain can be segmented according to the various orders of scale within the problem. According to Raynal \& Royon-Lebeaud (2007), three suitable scales can be defined by: the micro-scale (liquid films on packing), the meso-scale (an element of packing) and the macro-scale (whole reactor column). Simulations at each of these scales can be used to determine input parameters for each successive scale. This approach can reduce computational effort considerably.

Using this framework, this investigation focusses on the modelling of liquid films on structured packing. At these small scales the flow can be approximated by gravity-driven film-flow down an inclined plane. Film flow of 
this nature is not only important in the analysis of carbon capture, it is also important in many areas of engineering, such as falling film microreactors (Ho et al., 2011; Chasanis et al., 2010).

The efficiency of CO2 absorption within packed columns is dependent on the structure of the liquid films which form within the packing. In particular, the surface area and shape of the films have large impacts upon species absorption across the interface. Another significant contributory effect is the concentrations of species within the phases. Full-film flow (specific wetted area, $a=1$ ) is optimal in these situations because the surface area for absorption is maximised. Flow features, such as rivulets, can significantly reduce the surface area available for species absorption. Therefore, it is important to understand these flow features and develop ways to model them easily and accurately.

Many investigations detailed in the literature focus on two-dimensional simulations of liquid-film flow. Raynal et al. (2004) performed two-dimensional simulations of multiphase flow within structured packing to determine the liquid hold-up. This allowed them to make an approximation of the wet pressure drop within packed columns by combining data with the results of three-dimensional single-phase pressure drop calculations. Szulczewska et al. (2003) used two-dimensional VOF simulations to study the dependency of the interfacial area on flow parameters, such as the gas and the liquid flow rates. Haroun et al. (2010) used a modified volume-of-fluid (VOF) method to perform direct numerical simulation of gas-liquid flow on a small twodimensional section of structured packing. Species absorption across the interface and simple reaction kinetics were then included in the model to determine the effect of corrugation on the mass transfer rates.

Two-dimensional simulations can greatly simplify the approach and significantly reduce the computational effort required during CFD simulations. During full-film flow this approach gives a good approximation of the hydrodynamics. However, as flow rates decrease, the surface area minimising effect of surface tension can cause the flow to form rivulets. These structures are three-dimensional in nature and therefore cannot be resolved using a two-dimensional approach. It is important that these structures are resolved considering that they can significantly reduce the interfacial surface area, impacting heavily on mass transfer rates.

Rivulets have been modelled using three-dimensional simulations of multiphase flow using the VOF method (Hoffmann et al., 2005, 2006). It was shown that the the choice of boundary conditions and geometry is very im- 
portant to determine physically accurate results using CFD simulations. It was shown by Iso \& Chen (2011) that it is also important to consider the time-dependence of the phenomena when performing CFD simulations of multiphase flows. Three-dimensional, isothermal, non-reacting VOF simulations were performed to determine liquid film flow on an inclined steel plate. The specific wetted area of the plate was determined as the liquid flow rate was increased. This was repeated as the liquid flow rate was decreased back to the initial flow rate. A hysteresis pattern was observed in the wetted area as the flow rate was decreased, emphasising the importance of using time-dependent simulations.

Lan et al. (2010) performed a parametric study using a combination of experimental and three-dimensional, isothermal CFD simulations. They studied thin film flow on inclined planes and determined the effects of surface tension, contact angle, film flow rate and inclination angle on the film velocity, width and thickness.

The interface between a gas and a liquid phase should, in theory, have a thickness in the order of the distance between molecules (Theodorakakos \& Bergeles, 2004). This scale is significantly smaller than can be resolved in VOF simulations, so the interface is at least spread over 1 cell width. However, numerical diffusion of the volume fraction widens the reconstructed interface to a thickness of a few cells. Therefore, it is obvious that in order to accurately resolve the interface the cells must be as small as possible in the interface region. Due to the changing nature of the interface, standard VOF requires highly refined grids throughout the domain to ensure proper resolution at the interface as it progresses through the domain. This can significantly affect the computational requirements.

An approach which attempts to address this problem is local adaptive mesh refinement (AMR). Local AMR allows for dynamic refinement of the mesh in regions of high error and un-refinement in regions of low error (Jasak, 1996). In terms of the VOF method, refinement is made at the interface in order to ensure that the mesh can accurately resolve it. As the interface moves through the domain, successive mesh refinement and un-refinement can take place. Refinement is achieved using the addition of computational nodes, known as h-refinement (Jasak, 1996).

The AMR approach has been used successfully in many different applications, from single-phase flows to multiphase flows. Jasak \& Gosman (2000) developed an AMR procedure based on a-posteriori error estimates and solution gradients. They used this approach to solve supersonic flow of an ideal 
gas flowing over a forward facing step resulting in strong shock wave formations. Shock waves exhibit large gradients and so require high cell densities to be resolved accurately. Jasak \& Gosman showed that the use of AMR was able to suitably resolve these shocks.

In terms of multiphase flows, Theodorakakos \& Bergeles (2004) evaluated the effectiveness of AMR with the VOF method on various test cases. Simulations were performed on the convection of bubbles under prescribed flow velocities and droplet impact was also studied. They concluded that the AMR approach was able to reduce the computational time for simulations, whilst gaining very good accuracy of the interfacial region. In particular, they observed that a very small transition region between the gas and the liquid phases could be achieved, which is physically more realistic. Numerical diffusion of the interface was reduced, which addresses one of the disadvantages of the VOF method.

There have also been investigations using the VOF approach with AMR to study droplet impact onto thin liquid films (Brambilla \& Guardone, 2013; Nikolopoulos et al., 2007). This approach is particularly useful in these situations because of the range of scales observed. For example, during the breakup of the crown of fluid formed after impact. These examples show that significant improvements can be made to the VOF method with the inclusion of local AMR.

In terms of packed columns, surface texture on the structured packing has been shown to increase the wetted area (Iso \& Chen, 2011; Cooke et al., 2012), as well as impact on the structure of the films (Gu et al., 2004). In theory, this should enhance heat and mass transfer across the interface. This has been confirmed by Kohrt et al. (2011) who performed an experimental investigation to determine liquid-side mass transfer during flow over an inclined plate. The addition of a textured surface, typically found in commercial structured packing, to a smooth plate increased the mass-side transfer by as much as $80 \%$. However, the absorption of species across an interface is also dependent on the concentrations of those species within the phases. It has been shown that certain surface textures can cause regions of recirculation in the film ( $\mathrm{Gu}$ et al., 2004; Valluri et al., 2005). These regions of stagnant fluid will reduce the efficiency of CO2 absorption.

These investigations show the importance of surface texture in structured packing. This also raises the question of grid refinement at the surface of the packing. If these textures have such an influence on the flow dynamics, then it is reasonable to assume that grid densities must be high enough to resolve 
these effects. Therefore, it is important to consider whether local AMR at the interface is adequate in these situations.

This paper uses an open-source CFD solver, OpenFOAM (OpenCFD, 2011; OpenCFD., 2011) to study three-dimensional, two-phase, isothermal film flow using the VOF method. The first section of this paper examines gravity-driven film flow over a smooth inclined plane using a static grid (Cooke et al., 2012). The results are validated by comparison with experimental data from the literature and the Nusselt theory. The second part of this paper uses the local AMR approach in an effort to improve upon the results gained using a static grid. Improvements are expected in terms of simulation accuracy and computational effort.

\section{Numerical Methodology}

\subsection{Modelling and Governing Equations}

Due to the complex flow behaviours through packed columns and the multiphase, multiscale nature of the system it is inherently difficult to utilise CFD to simulate these reactors. In the literature to date, this has only been achieved by introducing simplifications to the model or by segmenting the problem into various scales (Raynal \& Royon-Lebeaud, 2007). This investigation simplified the problem by approximating the flow over the packed columns as gravity-driven flow down an inclined plane (Cooke et al., 2012). Species absorption and reaction kinetics were neglected in order to further simplify the modelling approach.

This investigation made use of the CFD software, OpenFOAM (OpenCFD, 2011; OpenCFD., 2011), a set of c++ libraries used to solve fluid flow problems. In particular, the solver interFOAM, which implements the VOF method (C.W. Hirt and B.D. Nichols, 1981) was used. This approach solves a single momentum equation for the fluid mixture, instead of one for each phase. A linear combination of the properties of the individual phases is used to derive the fluid properties of this mixture. The interface between the phases is resolved using an interface capturing technique, achieved by solving a transport equation for the volume fraction.

The volume fraction, $\alpha_{i}$ specifies the volume of the $i^{t h}$ phase within each computational cell and satisfies, $0 \leq \alpha_{i} \leq 1$. A cell completely filled with the $i^{\text {th }}$ phase has $\alpha_{i}=1$ and a cell devoid of the $i^{\text {th }}$ phase has $\alpha_{i}=0$. The interface is constructed using the values of $\alpha_{i}$ across the domain. For a twophase system, as is the case in this paper, the volume fraction need only to 
be determined for one phase (the liquid phase) because the volume fraction of the secondary phase is trivial, $\alpha_{2}=1-\alpha_{1}$.

Fluid properties such as the density, $\rho$ and the dynamic viscosity, $\mu$ are defined in each cell as a function of the volume fraction and are given by:

$$
\begin{aligned}
& \rho=\alpha_{1} \rho_{1}+\left(1-\alpha_{1}\right) \rho_{2}, \\
& \mu=\alpha_{1} \mu_{1}+\left(1-\alpha_{1}\right) \mu_{2},
\end{aligned}
$$

where the subscripts relate to the individual properties of each phase.

The volume fractions throughout the domain are determined by solving transport equations for $\alpha_{i}$. The transport equation for $\alpha_{1}$ used in the interFOAM solver is defined by (Rusche, 2002):

$$
\frac{\partial \alpha_{1}}{\partial t}+\nabla \cdot\left(\vec{v} \alpha_{1}\right)+\nabla \cdot\left(\overrightarrow{v_{r}} \alpha_{1}\left(1-\alpha_{1}\right)\right)=0,
$$

where $\overrightarrow{v_{r}}$ is a suitable velocity field selected to compress the interfacial region (Berberović et al., 2009). Equation 3 is the continuity equation for $\alpha_{1}$, with the notable addition of the $3^{\text {rd }}$ term. As described by Rusche (2002), this is an artificial compression term used to provide interface compression without need to use a compressive differencing scheme, with the advantage that the solution for $\alpha_{1}$ is bounded between 0 and 1, as required by the definition of volume fraction. Due to the form of this extra term it is only significant in the interfacial region, where it is required, and so has little effect on the solution throughout the rest of the domain.

Due to the low liquid film Reynolds numbers encountered in the simulations in this paper $\left(R e_{l}<230\right)$ the flow can be considered laminar. In the laminar regime the momentum equation is given by:

$$
\frac{\partial(\rho \vec{v})}{\partial t}+\nabla \cdot(\rho \vec{v} \vec{v})-\nabla \cdot \mu \nabla \vec{v}=-\nabla p+\rho \vec{g}+\vec{F}_{s}
$$

The contribution to the balance of momentum from surface tension, $\vec{F}_{s}$ is modelled using the continuum surface force (CSF) model (Brackbill et al., 1992) and is given by:

$$
\overrightarrow{F_{s}}=\sigma k(\vec{x}) \vec{n}
$$

where $\sigma$ is the surface tension, $\vec{n}$ is the unit normal vector to the interface and $k(\vec{x})$ is the curvature of the interface, as given by:

$$
\vec{n}=\frac{\nabla \alpha}{|\nabla \alpha|}
$$




$$
k(\vec{x})=\nabla \cdot \vec{n} .
$$

In cells adjacent to a wall boundary the definition of the unit normal vector, $\vec{n}$, is adjusted in order to take account of the liquid contact angle. The method in this paper used a constant wall contact angle, $\theta_{w}$ and the unit normal vector is defined as (Gu et al., 2004):

$$
\vec{n}=\overrightarrow{n_{w}} \cos \left(\theta_{w}\right)+\overrightarrow{m_{w}} \sin \left(\theta_{w}\right),
$$

where $\overrightarrow{n_{w}}$ is the unit vector normal to the wall and $\overrightarrow{m_{w}}$ is the unit vector tangential to the wall.

\subsubsection{Adaptive Grid Refinement}

Adaptive grid refinement is a method used to dynamically alter the mesh density throughout the domain. For example, in regions of high gradients, large numbers of cells are required to accurately resolve the solution. In principle, this can be achieved by producing an initial grid whereby mesh density is high in these specific regions, whilst being of low density in regions of less importance in order to minimise the run-time of simulations.

In the VOF method it is important to accurately resolve the interfacial region between two phases. In this method the interface is spread over a few cells, due to numerical diffusion in the transport equation for volume fraction. The accuracy of the interface can be improved by using high densities of cells around the interfacial region. However, the location of the interface is not known a priori and so using a static grid method would require a high density mesh throughout the domain, creating excessive simulations run-times.

Adaptive Mesh Refinement (AMR) allows one to initiate the simulation using a relatively coarse grid, which is then successively refined and unrefined according the specific location of the interface. This enables accurate resolution of the interface, whilst keeping run-time to a minimum. The only overhead is created by the process of mesh adaptation.

In this investigation the solver interDymFoam was used for the AMR simulations. This is an extension of the standard VOF solver, interFoam, to include dynamic manipulation of the computational grid. A dictionary file is used to specify the parameters for the grid manipulation. Grid refinement can be performed at a specific time-step interval and based on specific numerical fields. The initial grid is refined in regions where this field lies within a specific range. These cells are then un-refined back to the initial mesh when the value of the field moves outside of this range. The refinement of the 
mesh is performed by adding computational nodes along the mid-points of cells within the specified refinement regions (Brambilla \& Guardone, 2013). Hence, a 3-dimensional hexahedral cell will be split into 8 new cells. The solver limits the number of cell divisions of the initial mesh to a predetermined value.

\subsubsection{Important Parameters}

The liquid film Reynolds number, $R e_{l}$ is an important non-dimensional parameter in the analysis of liquid-film flow and is defined by (Iso \& Chen, 2011):

$$
R e_{l}=\frac{\rho_{l} \cdot V_{l} \cdot \delta}{\mu_{l}},
$$

where $V_{l}$ is the average liquid film velocity defined as

$$
V_{l}=\frac{Q_{l}}{\delta \cdot w}
$$

$Q_{l}$ is the volumetric flow rate of the liquid and $w$ is the width of the film, which is approximated as the full plate width during these simulations. The liquid-film thickness, $\delta$ is given by:

$$
\delta=\left[\frac{3 \cdot \mu_{l} \cdot Q_{l}}{\left(\rho_{l}-\rho_{g}\right) \cdot g \cdot \sin \theta \cdot w}\right]^{\frac{1}{3}},
$$

where $\theta$ is the plate inclination angle and the subscripts, $l$ and $g$ refer to the liquid and gas phases, respectively.

Another important parameter used in this investigation is the specific

wetted area of a plate, $a$. It is calculated as the ratio of the wetted area to the total area of the plate,

$$
a=\frac{A_{w}}{A_{t}} .
$$

The value of the specific wetted area lies in the range $0<a<1$, where $a=1$ represents a fully wetted plate.

\subsection{Computational Domain}

The computational domain was chosen to be a smooth, inclined steel plate bordered by steel walls at the sides (see Fig. 1)(Hoffmann et al., 2005, 2006; Iso \& Chen, 2011). This domain is an approximate representation of a small section of structured packing. The plate dimensions were $0.06 \mathrm{~m} \times$ 


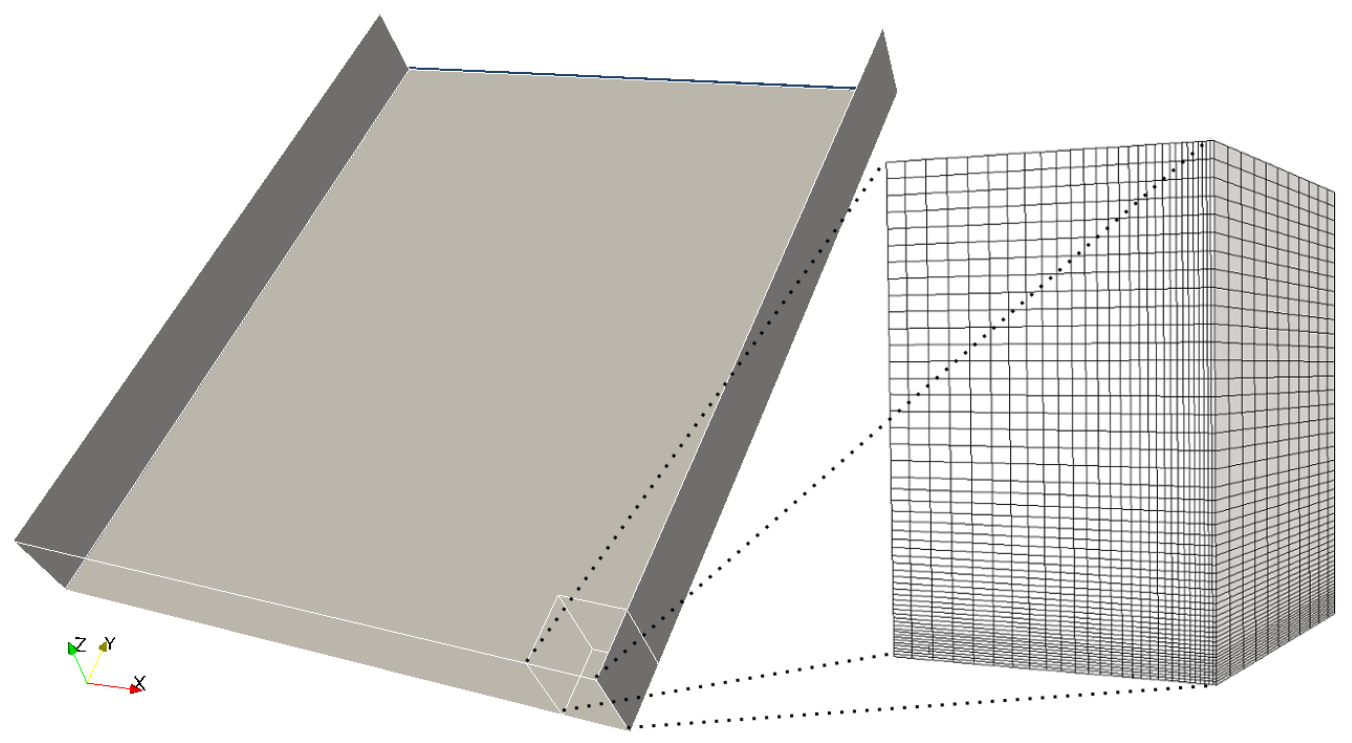

Figure 1: Computational domain and very fine static mesh

$0.05 \mathrm{~m} \times 0.007 \mathrm{~m}$ (height $\times$ width $\times$ depth $).$ The depth of liquid at the inlet was selected to be $0.4 \mathrm{~mm}$ across the full width of the plate, in accordance with previous investigations in the literature (Hoffmann et al., 2005, 2006; Iso \& Chen, 2011).

This investigation used two different approaches to the meshing procedure (see Table. 1). Firstly, simulations were run using a structured non-uniform hexahedral static mesh, whereby mesh independence checks (see appendix Appendix A) were performed to determine an adequately fine grid resolution (Cooke et al., 2012). The mesh is shown in Fig. 1 and consisted of 1.0 million cells.

Secondly, simulations were run using the local AMR method, using two different conditions on the refinement (see Table 1). Refinement around the interface with liquid-phase volume fractions in the range, $0.2<\alpha<0.8$ is denoted as the partial-film mesh, whereas refinement around the interface and the whole film region with liquid-phase volume fractions in the range, $0.2<\alpha<1.0$ is denoted as the full-film mesh. An initial mesh was selected with 192000 structured non-uniform hexahedral cells. A grid independence study was performed to determine an adequate limit on the number of cell 

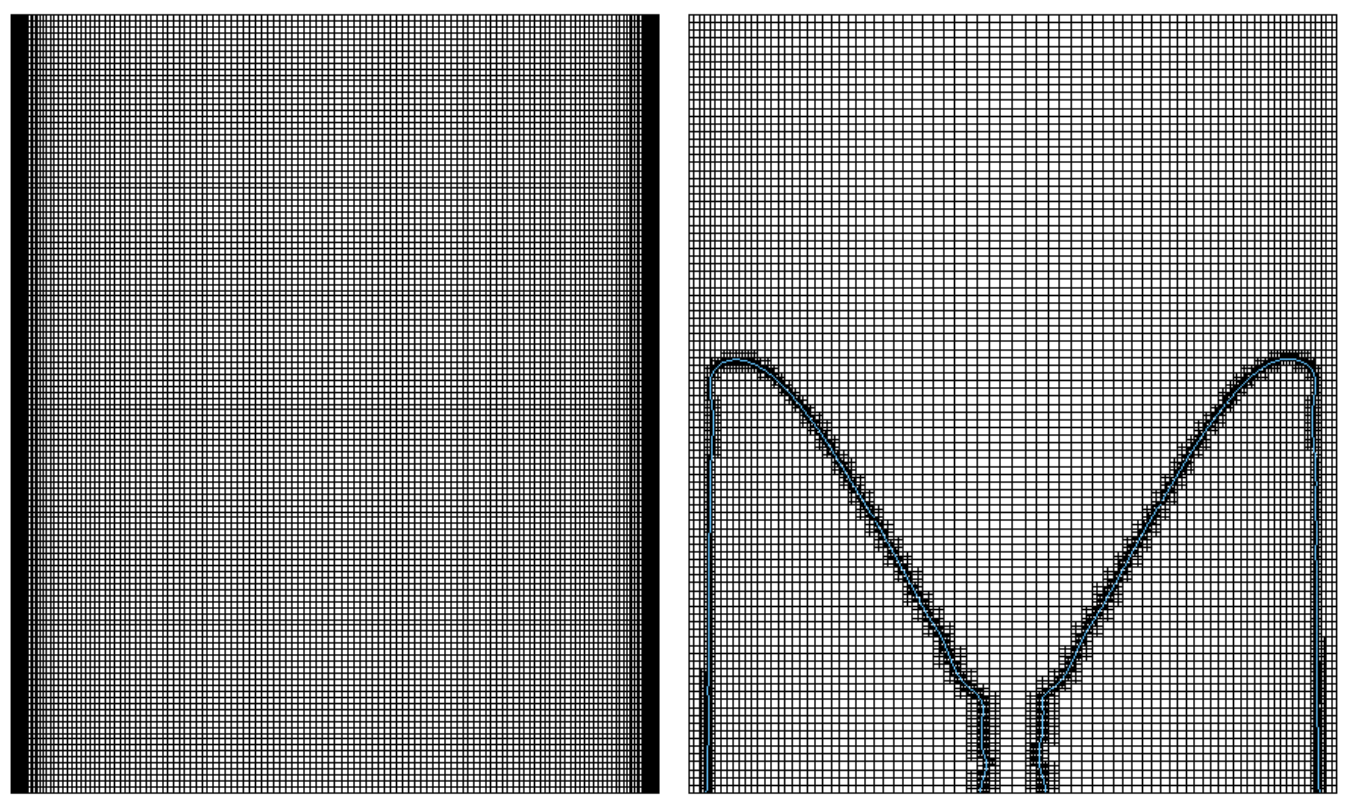

Figure 2: Comparison of the static mesh with the partial-film AMR mesh (blue line is the gas-liquid interface)

divisions. Limits of 2 and 3 were tested and the limit of 2 was found to be most appropriate. The extra level of refinement created a much larger number of cells within the whole domain causing the run-time to increase approximately 20 times. However, the difference in the calculated specific wetted area was only $2.72 \%$.

Figure 2 shows a comparison of the mesh used in the static grid and partial-film AMR grid simulations. The sections of mesh were selected from the plate surface at time, $t=0.36 \mathrm{~s}$ with $R e_{l}=156.85$. On the left, it can be seen that the static mesh is finer throughout the whole domain, whereas on the right it can be seen that the AMR mesh is fine just around the gas liquid interface, denoted by the blue line. Figure 3 shows a closer view of the static mesh and the AMR mesh. Here it can be seen that the static mesh is highly refined throughout the domain, even in regions where it is not required. On the other hand, the AMR mesh is much more highly refined at the interface, where high grid density is required for accurate resolution of the interface. In other parts of the domain, the mesh is coarser, which helps to maintain reasonable simulation run-times. 

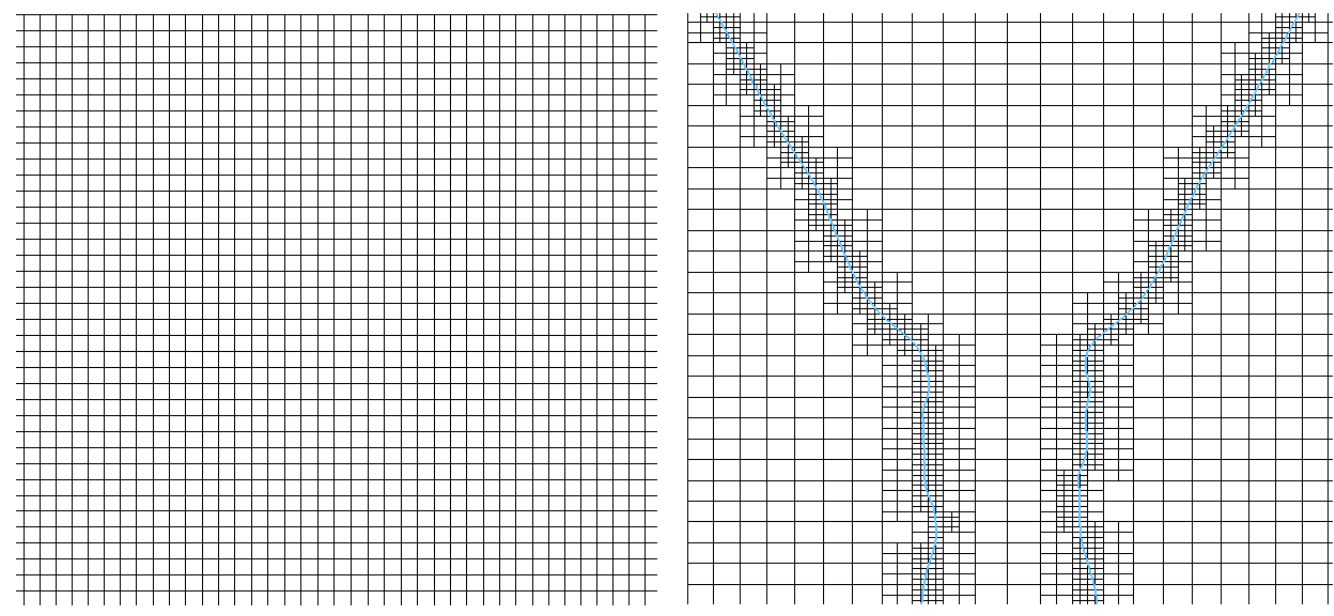

Figure 3: Closer view of comparison of the static mesh with the partial-film AMR mesh (blue line is the gas-liquid interface)

Table 1: Computational Meshes

\begin{tabular}{ccc}
\hline Mesh Name & $\begin{array}{c}\text { Initial Mesh } \\
\text { Density }\end{array}$ & $\begin{array}{c}\text { Refinement } \\
\text { Conditions }\end{array}$ \\
\hline Static Mesh & $1.0 \times 10^{6}$ & - \\
Partial-Film & $1.92 \times 10^{5}$ & $0.2<\alpha<0.8$ \\
Mesh & & $0.2<\alpha<1.0$ \\
Full-Film & $1.92 \times 10^{5}$ & \\
Mesh & &
\end{tabular}

\subsection{Simulation Set-Up}

For all simulations a constant inclination angle of $\theta=60^{\circ}$ was chosen to allow comparisons with current data in the literature. A range of $R e_{l}$ were tested by suitably altering the velocity of the liquid film at the inlet. During this investigation counter-current flow of gas was neglected to again allow comparison with previous data in the literature. As mentioned previously, the simulations were treated as laminar because of the low liquid film Reynolds numbers encountered, $R e_{l}<230$.

Time-dependent simulations were carried out using a variable time-step to ensure a Courant number below 1.0, resulting in time-steps of approximately $1 \times 10^{-5} \mathrm{~s}$. Simulations were allowed to run until a steady or pseudo-steady 
Table 2: Phase Properties

\begin{tabular}{ccc}
\hline Phase & $\mu[\mathrm{Pa} \cdot \mathrm{s}]$ & $\rho\left[\mathrm{Kg} \cdot \mathrm{m}^{-3}\right]$ \\
\hline Liquid & $8.899 \times 10^{-4}$ & 997 \\
Gas & $1.831 \times 10^{-5}$ & 1.185
\end{tabular}

state was reached. This was assured by monitoring the specific wetted area of the plates as a function of time. During the AMR simulations the mesh adaptation was performed every 5 time steps.

The dynamic viscosity and density of the constituent phases are given in Table 2. The surface tension was set to $\sigma=0.0728 \mathrm{~N} \cdot \mathrm{m}^{-1}$ and a static contact angle of $\theta_{w}=70^{\circ}$ was selected.

\section{Standard Grid Refinement}

\subsection{Results \& Discussion}

In accordance with Cooke et al. (2012), the numerical methods used throughout the investigation were validated by comparison with experimental data from the literature and with the predictions of the Nusselt theory. Using the static grid, simulations were performed to determine the specific wetted area across a range of liquid film Reynolds numbers and comparisons were made with existing experimental data (Hoffmann et al., 2005, 2006). Figure 4 shows a plot of the simulated data and the comparative experimental data.

The Nusselt theory (Nusselt, 1916) provides a theoretical prediction of the velocity profile within a liquid film during gravity-driven flow down an inclined plane. This prediction was used to provide further validation of the computational approach used throughout this paper. The simulation was performed using the static grid at $R e_{l}=224$ and inclination angles of $\theta=30^{\circ}$ and $\theta=60^{\circ}$ (Cooke et al., 2012). At this liquid film Reynolds number a full film had developed across the plate allowing the effects of rivulets to be neglected, considering these flow formations are not considered in the Nusselt theory. The film velocity profile was extracted from a central position on the plate in order to minimise the side-wall effects, which are also not considered in the Nusselt theory. A position $0.5 \mathrm{~mm}$ above the outlet (far from the inlet) was selected to ensure that the film was fully developed. Figure 5 shows the comparison between the predictions of the Nusselt theory and the data obtained using CFD simulations. 


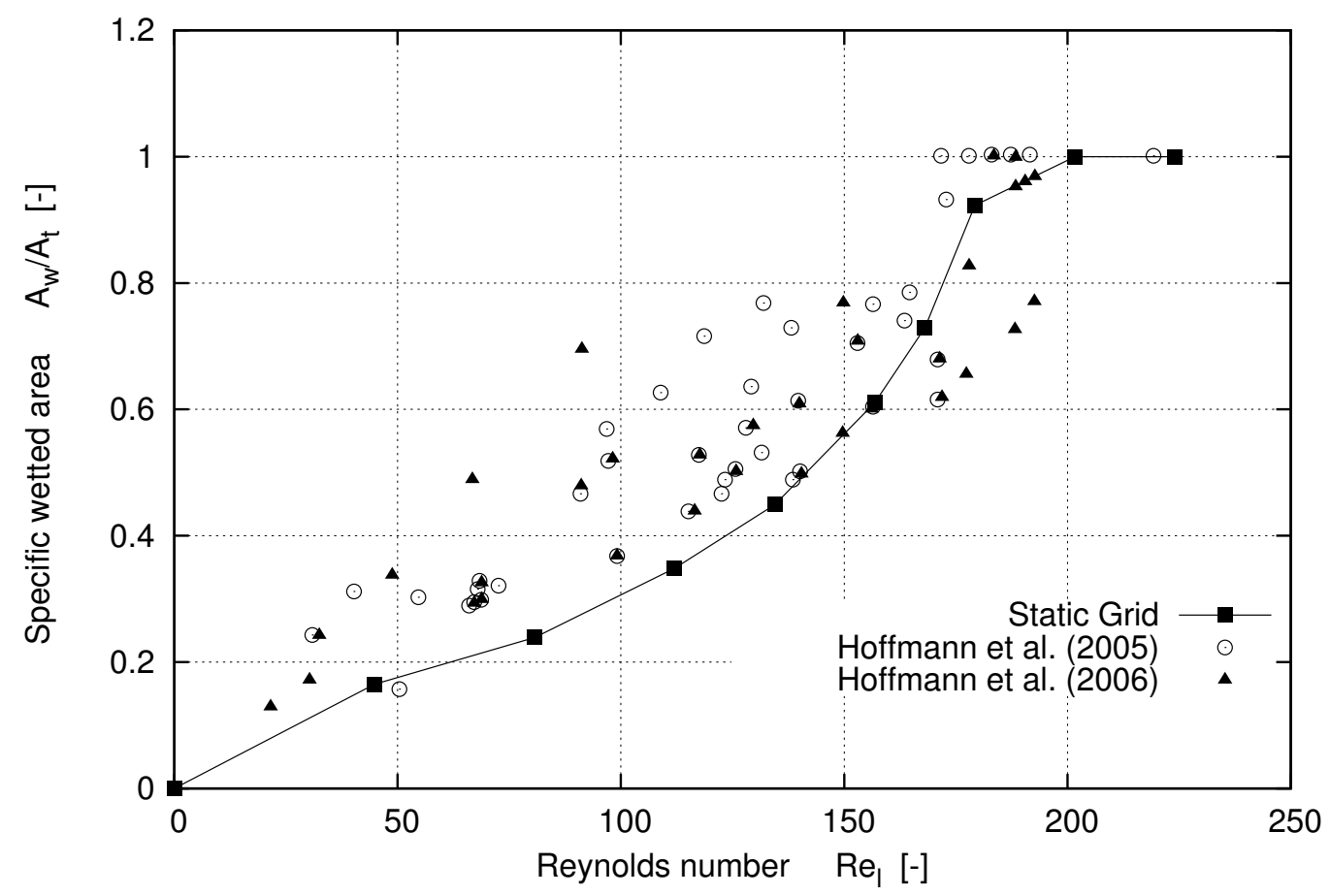

Figure 4: The specific wetted area against $R e_{l}$ for the static grid

It is observed in fig. 4 that good agreement with experimental data is achieved at higher values of $R e_{l}$, which is further confirmed by the predictions of Nusselt theory in fig. 5. However, at lower $R e_{l}$ the wetted area is underpredicted. Comparison of the results were made with other CFD data from the literature, along with the experimental data of Hoffmann et al. (2005, 2006) (see fig. 6). Improvements in accuracy have been made in comparison to other simulations in the literature, possibly due to the finer grid resolutions used in this paper. Since this improvement was observed, it was postulated that further improvements could be made by increasing the grid resolution at the interface by using AMR. This should increase the accuracy of the interface reconstruction, which should improve simulations at lower $R e_{l}$, where the curvature of the interface is larger. This will be investigated throughout the rest of this paper. It is also important to consider that inaccuracies in the simulations may result from fundamental inefficiencies of the VOF method. However, if this is the case, extensive research may need to be performed to 


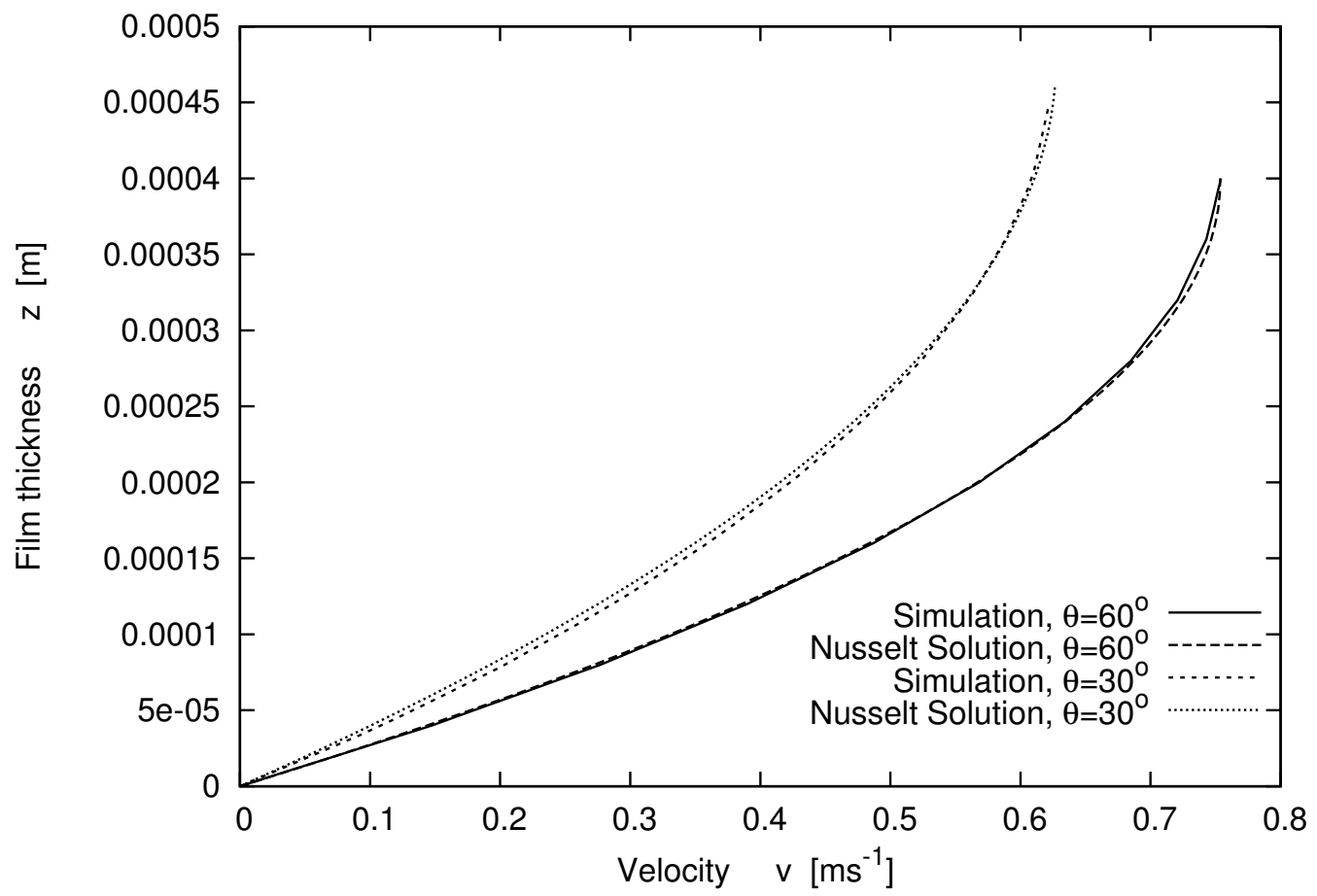

Figure 5: Comparison of film velocity profile against the Nusselt solution at $R e_{l}=224$

develop alternative, novel approaches.

\section{Adaptive Grid Refinement}

\subsection{Refinement at the Interface}

It was postulated that the differences between the data of CFD simulations and real-world data, especially at lower $R e_{l}$, were caused by inefficiencies of the VOF method. Since some improvement was observed by using a higher density grid resolution across the domain, it was assumed that further improvement could possibly be made by increasing the grid resolution at the interface. This should increase the accuracy of interface reconstruction. Local AMR was used to achieve the desired increase in grid resolution at the interface, whilst maintaining reasonable run-times by using a coarse mesh in regions of less physical interest. 


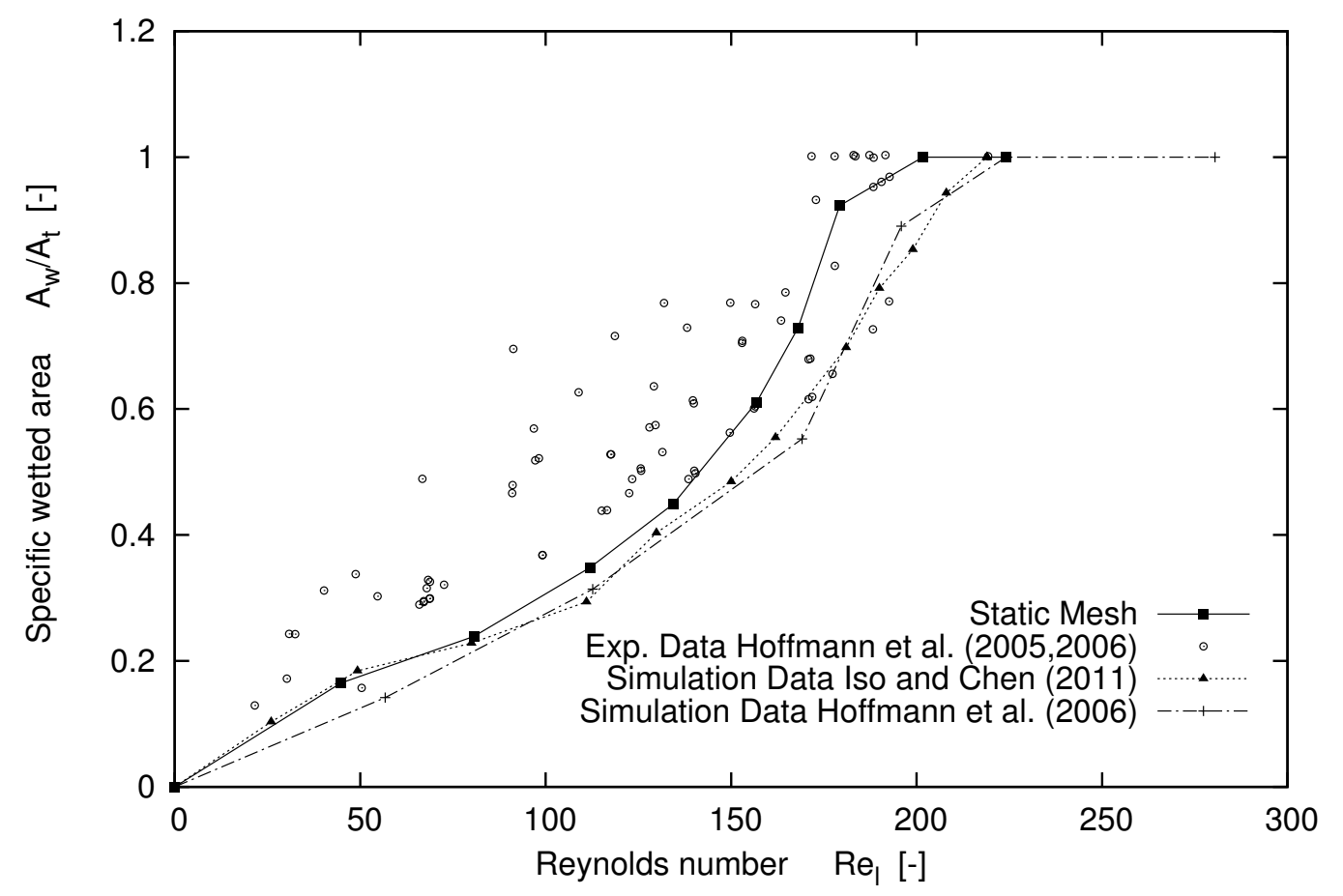

Figure 6: Comparison of Static Grid Simulation with Literature

\subsection{Results}

In order to assess the improvements made by using local AMR at the gasliquid interface, the wetted area against $R e_{l}$ was plotted (see Fig. 7). Figure 7 also displays the experimental data of Hoffmann et al. $(2005,2006)$ and the CFD data obtained using the static grid from section 3.1. It is noted that the results from local AMR were improved at the lower and higher ranges of liquid film Reynolds numbers. An important observation is the fact that local AMR provides results in much greater correlation with experimental data in the range $50<R e_{l}<100$. In this region the specific wetted area initially falls before rising again, a behaviour which is expressed in the experimental data. Simulations with a highly refined static mesh were unable to replicate this behaviour, indicating the importance of using local AMR at lower $R e_{l}$.

It would be expected that as the liquid film Reynolds number increases the wetted area would also increase. However, as noted previously, it can be seen that between $R e_{l}=44.8$ and $R e_{l}=58.3$ the wetted area plateaus, falling 


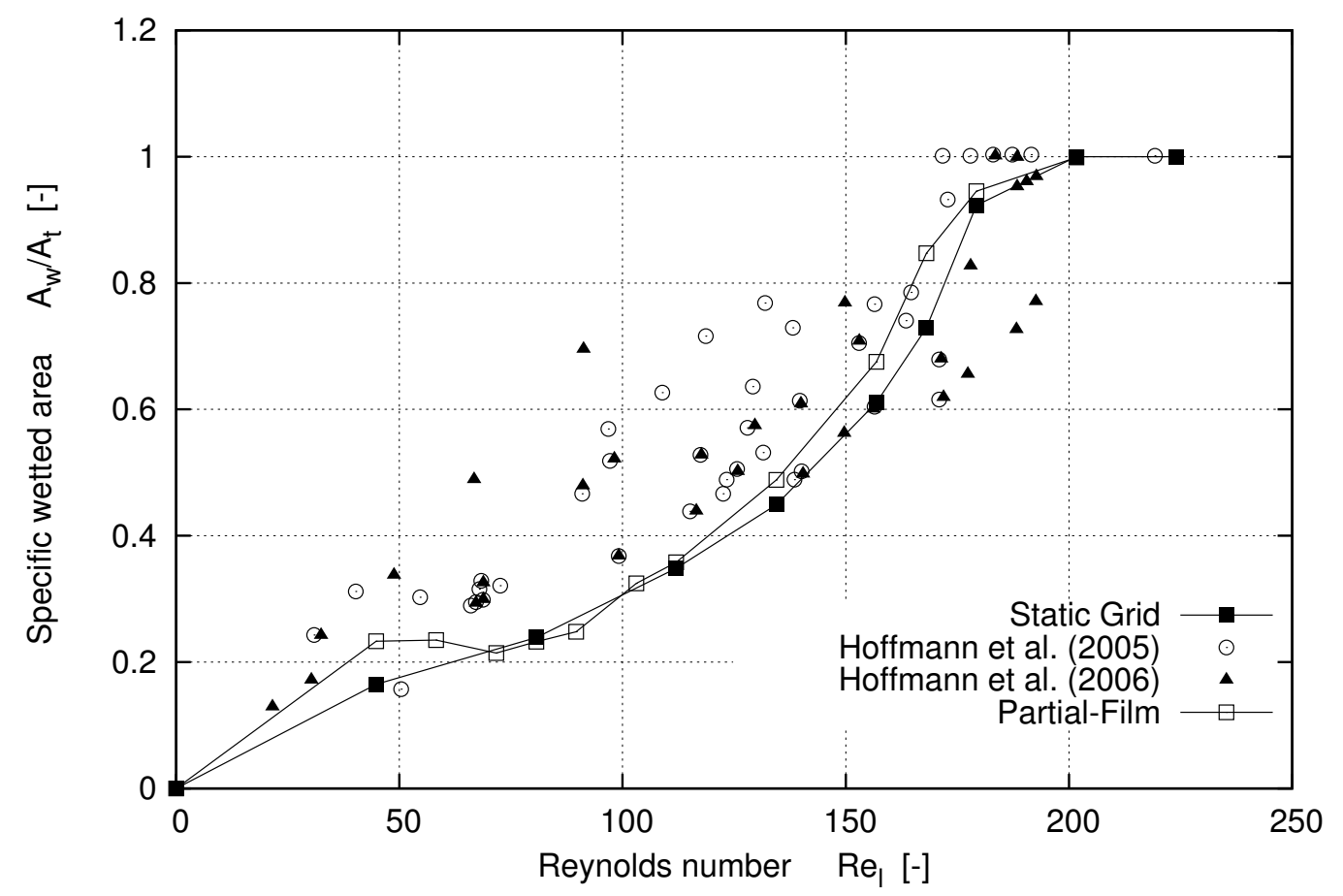

Figure 7: The specific wetted area against $R e_{l}$ for AMR at the interface

at $R e_{l}=71.7$ and then rising in the assumed manner as further increases in $R e_{l}$ are made. This can be explained by the flow phenomena observed at each of these $R e_{l}$. At $R e_{l}=44.8$, the flow forms 4 rivulets (see Fig. 8), reducing to 3 rivulets at $R e_{l}=58.3$ (see Fig. 9) and forming a single rivulet at $R e_{l}=71.7$ (see Fig. 10). At these lower $R e_{l}$, the increasing flow rate changes the behaviour of the flow. As the rivulets combine, the wetted area stays constant, or even falls, due to the surface area minimising effect of surface tension. It can be assumed that the $R e_{l}$ where a single rivulet is formed is in the region $58.3<R e_{l}<71.7$, which may explain the cluster of experimental points in this range (see Fig. 7).

By comparing the interface contours of simulations using the static grid and the partial-film grid it is possible to see why the static grid underestimates at lower $R e_{l}$ and does not correlate properly with the experimental data. Fig 11 shows the interface contours at $R e_{l}=44.8$. It can be seen that the static grid only resolves a single rivulet, whilst the partial-film grid 


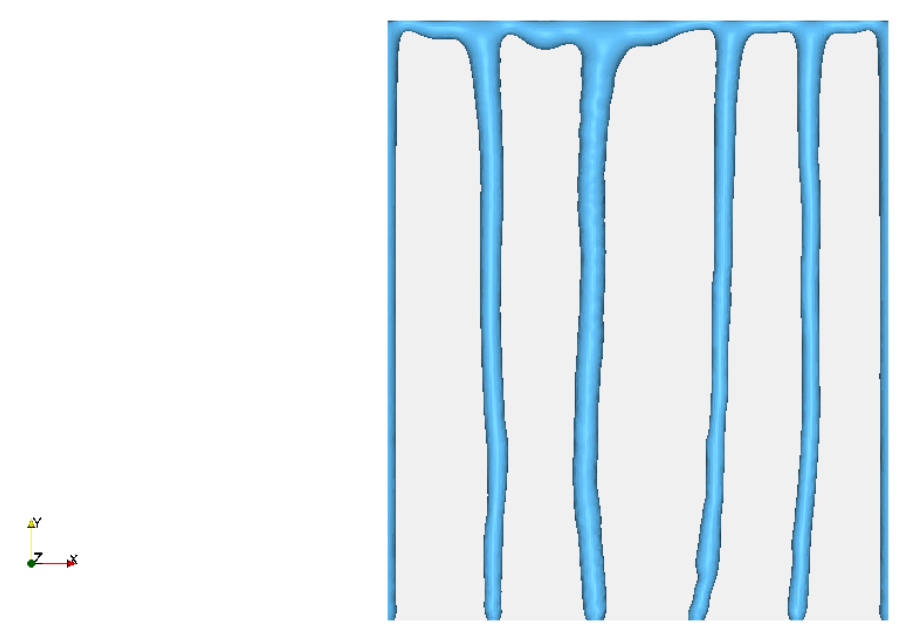

Figure 8: Contour plot of gas-liquid interface at $R e_{l}=44.8$ (Partial-film mesh)

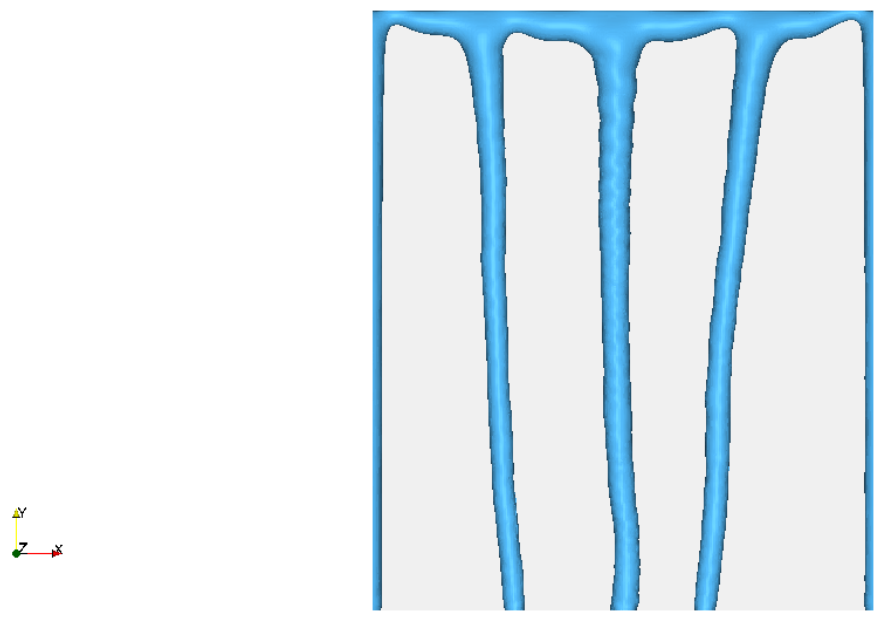

Figure 9: Contour plot of gas-liquid interface at $R e_{l}=58.3$ (Partial-film mesh)

resolves 4 . Due to the surface area minimising effect of surface tension, a single rivulet would have a smaller wetted area than 4 rivulets, as observed from the data.

The experimental data in fig. 7 is quite scattered and so it is difficult to make direct correlations between the simulations and experimental data. However, the explanation of the local maximum between $50<R e_{l}<100$, 


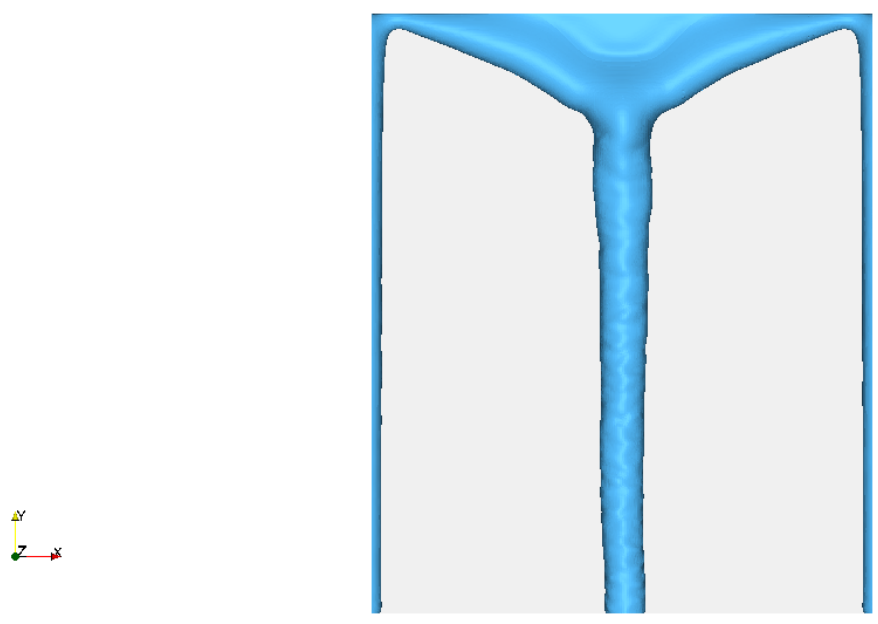

Figure 10: Contour plot of gas-liquid interface at $R e_{l}=71.7$ (Partial-film mesh)
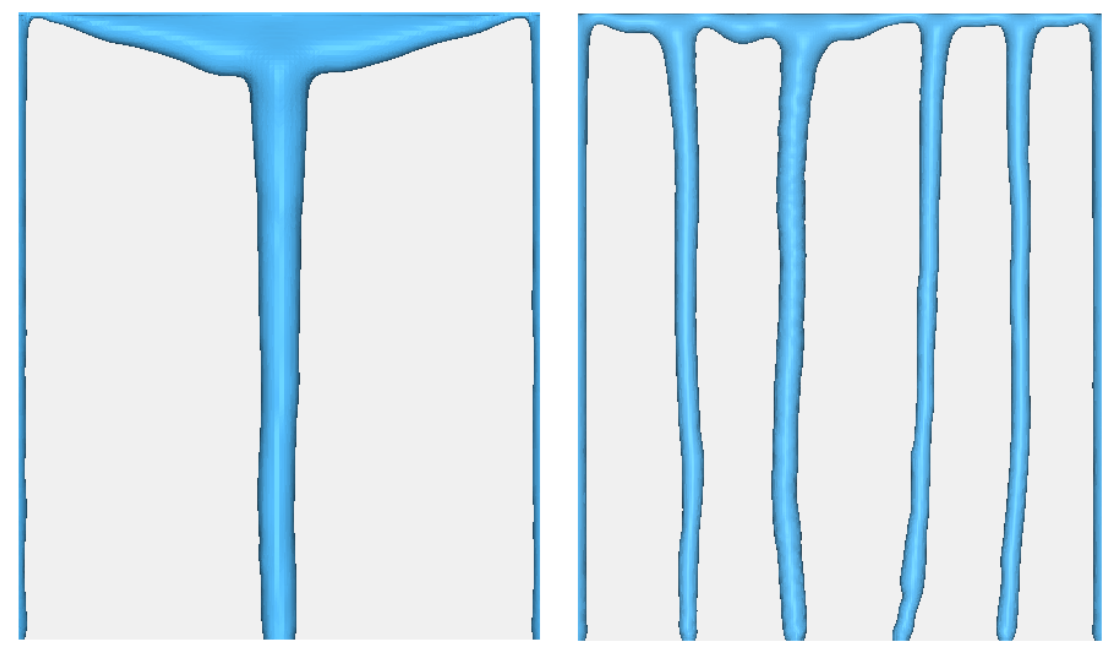

Figure 11: Contour plot of gas-liquid interface at $R e_{l}=44.8$ for static grid (left) and Partial-film grid (right)

using the surface contour plots above, helps to strengthen this assumed correlation. 


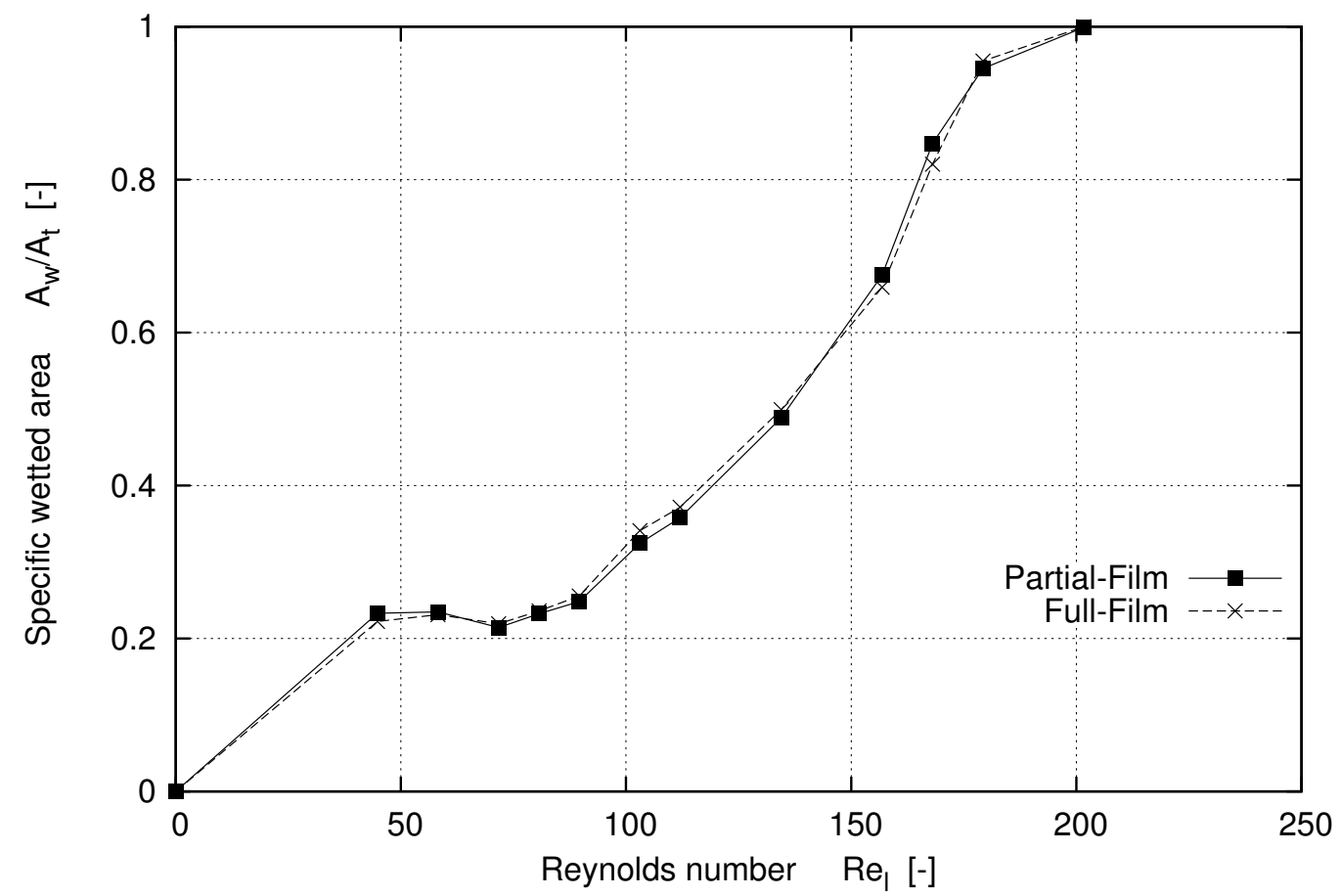

Figure 12: The specific wetted area against $R e_{l}$ for various degrees of AMR

\subsection{Full-Film Refinement}

It has been shown that local AMR about the interface was more able to replicate the physical phenomena of the real-world flow. However, there was still an under-estimation of the wetted area at lower $R e_{l}$. It was considered that improvements may be made by refining the whole film, rather than just at the interface. The film within packed columns and also on these inclined plates is relatively thin and so shear layers at the wall may require extra refinement to be resolved accurately, especially if these shear layers have a significant impact on the interface, due to their close proximity.

AMR simulations were performed to determine the wetted area across a range of $R e_{l}$, refining the whole liquid film. Comparisons were then made with the results of AMR simulations with refinement only around the interface (see Fig. 12).

It is observed that the results using the two methods are fairly consistent across the whole range of $R e_{l}$ tested. Table 3 shows the run-times for each 
Table 3: Time to convergence

\begin{tabular}{cccc}
\hline$R e_{l}$ & $\begin{array}{c}\text { Convergence Time } \\
\text { [days] } \\
\text { Static Mesh }\end{array}$ & $\begin{array}{c}\text { Convergence Time } \\
\text { [days] } \\
\text { Partial-Film }\end{array}$ & $\begin{array}{c}\text { Convergence Time } \\
\text { [days] } \\
\text { Full-Film }\end{array}$ \\
\hline 44.81 & 0.14 & 1.75 & 1.90 \\
58.26 & - & 1.55 & 2.09 \\
71.70 & - & 1.52 & 2.47 \\
80.67 & 0.39 & 0.89 & 2.74 \\
89.63 & - & 0.85 & 2.23 \\
103.07 & - & 0.80 & 1.87 \\
112.04 & 0.15 & 0.84 & 1.94 \\
134.44 & 0.38 & 0.99 & 3.49 \\
156.85 & 0.16 & 0.56 & 1.50 \\
168.05 & 0.42 & 2.20 & 6.91 \\
179.26 & 0.53 & 1.98 & 9.60 \\
201.66 & 0.26 & 0.93 & -
\end{tabular}

simulation. Data that is not available is denoted by "_" in the table. The static mesh data was acquired from a previous paper by the authors and a larger range of $R e_{l}$ were tested in this investigation. For the full-film simulation data was not obtained for $R e_{l}=201.66$ due to excessive simulation run-times at these high $R e_{l}$.

Local AMR throughout the whole liquid film was shown to exhibit insignificant improvements. Run-times of the simulations were increased across the range of $R e_{l}$ tested, with large increases seen at higher $R e_{l}$. It is obvious that under the situations tested in this investigation, local AMR throughout the whole of the liquid film was an over-complication resulting in dramatically increased run-times, with negligible improvement in accuracy. Therefore, local AMR about the interface is optimal. This finding is also important when scaling up the problem to determine the hydrodynamics within larger sections of packing. In these situations an initial coarse grid can be selected and refinement made at the gas-liquid interface, ensuring that physically accurate 


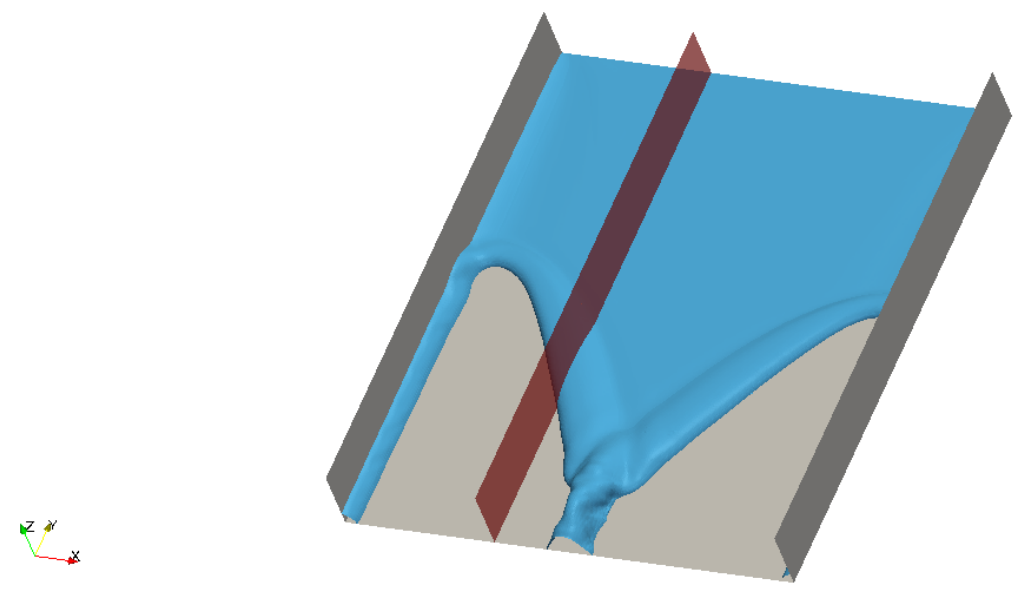

Figure 13: Cutting plane used for interface plots

results are achieved, whilst maintaining reasonable simulation run-times.

Despite the fact that the differences in specific wetted areas between the two types of AMR simulations was minimal, it was important to examine the structure of the liquid film to determine whether any major differences occurred. To determine the film depths down the plate a slice was taken $(x=0.017 \mathrm{~m})$, as shown in Fig. 13 .

Figure 14 shows a comparative plot of the film depths for the full- and partial-film simulations. It was observed that the full-film simulations were able to resolve ripples preceding the advancing front. These were damped out in the partial-film simulations. This could be due to the fact that any effect of the plate surface on the interface was more resolved using full-film refinement. This may be an important consideration to make when absorption is added to the model. Interfacial absorption is dependent on the shape of the interface and so the ripples observed may affect the absorption of CO2 across the interface. However, the observed differences in film interface structure are minimal and so may not have a significant impact on the absorption of species across it. When considering counter-current gas flow it has been observed that this can cause an increase in the thickness and fluctuation of liquid films (Xu et al., 2008). We are currently running counter-current flow simulations to determine if these differences in interface contour are observed.

Another important consideration needs to be made when chemical ab- 


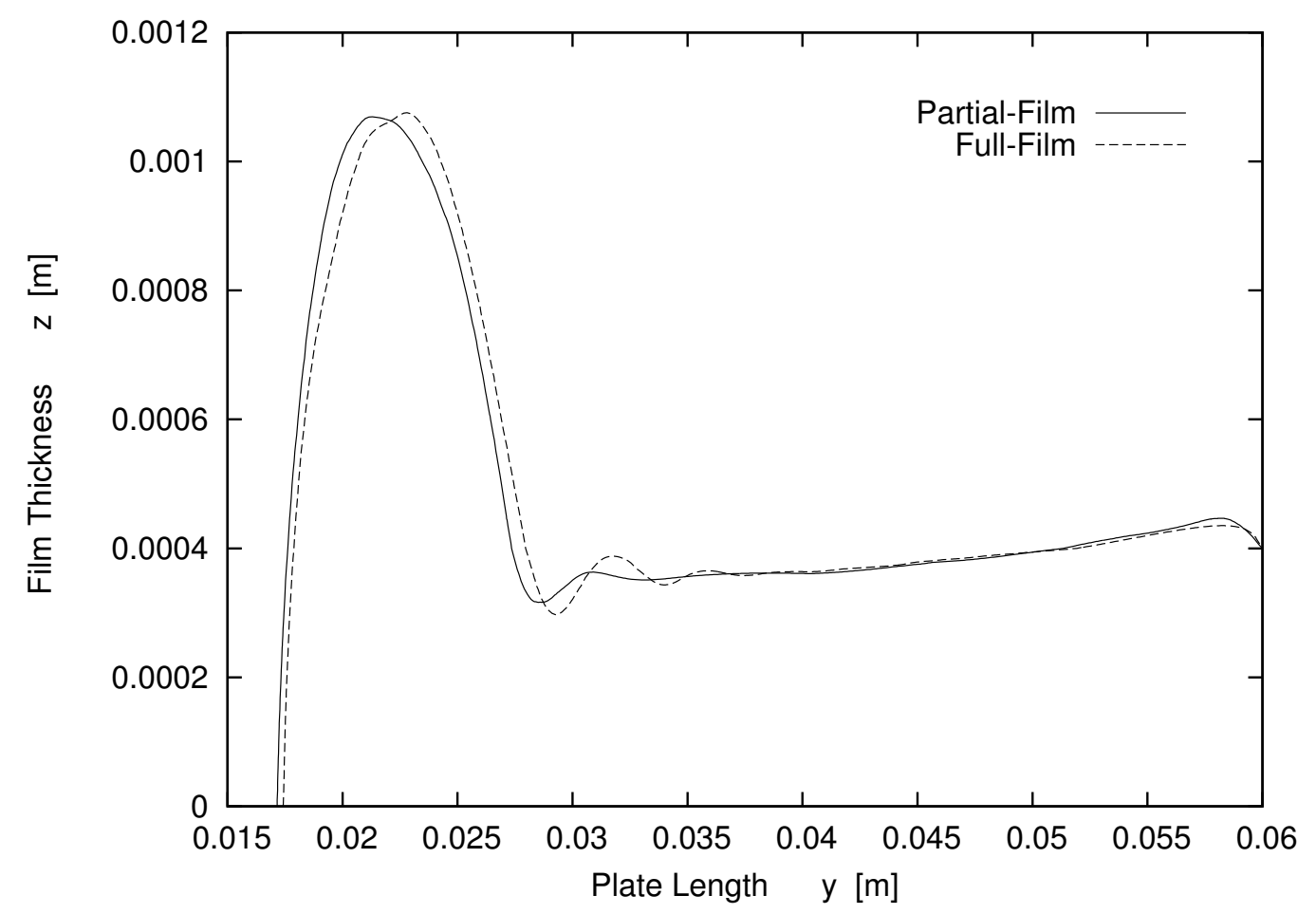

Figure 14: Interface plots

sorption and reaction kinetics are added to the computational models. In regions rich in MEA the reaction with $\mathrm{CO} 2$ would occur relatively quickly close to the interfacial region and so refinement at the interface would allow these reactions to be accurately resolved. However, as MEA becomes depleted at the interface, $\mathrm{CO} 2$ will diffuse further into the film, moving into un-refined regions. In these situations the reactions may not be accurately resolved and so it may be important for the whole film to be refined using local AMR. These considerations are out of the scope of this paper and will be analysed in future investigations.

\subsection{Improvements to Interface Refinement}

It can be seen in Table 3 that the Partial-film simulations using local AMR at the interface increased the run-times, especially at $R e_{l}=44.81$. These increases in run-time are acceptable considering the increases in accuracy gained. However, it was considered that improvements might be made on the run-times, whilst maintaining the desired accuracy. 
Table 4: Percentage changes from Partial-film to coarser Partial-film

\begin{tabular}{ccc}
\hline$R e_{l}$ & $\begin{array}{c}\text { Change in } \\
\text { Wetted } \\
\text { Area [\%] }\end{array}$ & $\begin{array}{c}\text { Change in } \\
\text { Run-time } \\
{[\%]}\end{array}$ \\
\hline 44.81 & -0.94 & -81.49 \\
156.85 & -3.96 & -74.49
\end{tabular}

Further simulations were run using a much coarser initial grid of only 32640 cells, in comparison to the 192000 used originally. An extra level of refinement was used at the interface to try to maintain the accuracy. Simulations were run at $R e_{l}=44.81$ and $R e_{l}=156.85$ using this new set-up.

Table 4 shows the percentage changes between the original Partial-film simulations and the coarser Partial-film simulations. There is only a small drop in the wetted areas, but a significant drop in the simulation run-times. This shows that the approach can be optimised to reduce the computational effort involved, whilst maintaining accuracy, by the choice of initial meshes and the number of refinement levels. This makes AMR approaches a promising option when the domain is scaled up to simulate larger sections of packed columns.

Another important consideration is the frequency of mesh updates. The process of updating the mesh creates a significant overhead, which takes of the order of a second to complete. Therefore, careful consideration of the required frequency of mesh updates may significantly further reduce simulation run-times.

\section{Conclusion}

In this investigation the VOF method, along with local AMR, was used to study isothermal, non-reacting, gravity-driven flow. The domain was chosen as an approximation to a small section of the packing material within packed columns used for CO2 absorption. Initially, simulations were performed using a static mesh method, whereby suitable refinement to the mesh was made via a mesh independence study. This highly refined mesh was able to improve upon previous studies in the literature and was more able to replicate experimental data. However, the specific wetted area was still under-predicted at smaller values of the liquid film Reynolds number. This approach would also 
have significant disadvantages when the domain is scaled up because there are large regions of high density mesh where it is simply not required.

Local AMR was used to try to improve the prediction of wetted area. AMR refinement of the gas-liquid interface significantly improved the computational results, especially at lower $R e_{l}$. The data was closer to experimental results and better replicated the trends observed at the lower range of $R e_{l}$. This approach has the advantage that mesh density is high in regions where it is required and lower in other regions. Therefore, this method may have better scalability and could be a viable approach to studying the hydrodynamics within larger sections of packing. However, under-predictions were still observed at lower $R e_{l}$. This may indicate a fundamental problem with using the VOF methodology for these types of thin-film simulations, since other authors' work also exhibit under-predictions at the lower range of $R e_{l}$. Taking this into consideration, we are currently investigating alternative approaches to thin-film modelling, which may improve upon these inaccuracies.

Finally, local AMR was used to refine the whole film region to examine whether the close proximity of the plate surface was affecting the results. It was found that simulation run-times were increased for all $R e_{l}$ tested, as would be expected. However, the computational results showed marginal differences and so it was concluded that local AMR should be applied at the interface only. Also, at larger $R e_{l}$ the run-times were significantly increased as the volume of the liquid film became larger. This shows that this approach would encounter difficulties scaling to larger domains, such as larger sections of structured packed.

Future investigations will focus on the development of alternative approaches to thin-film modelling as well as the addition of chemical absorption and reaction kinetics into the model. This will allow the authors to test the accuracy of local AMR at the interface with the inclusion of reactions and to assess the validity of this method for simulating packed column reactors for CO2 absorption. Also, the simulations will be scaled-up so that meso-scale sections of packing can be analysed. This will include optimisation of the initial mesh density, the number of refinement levels and the frequency of mesh refinements in order to keep run-times to a minimum, whilst maintaining good accuracy. 


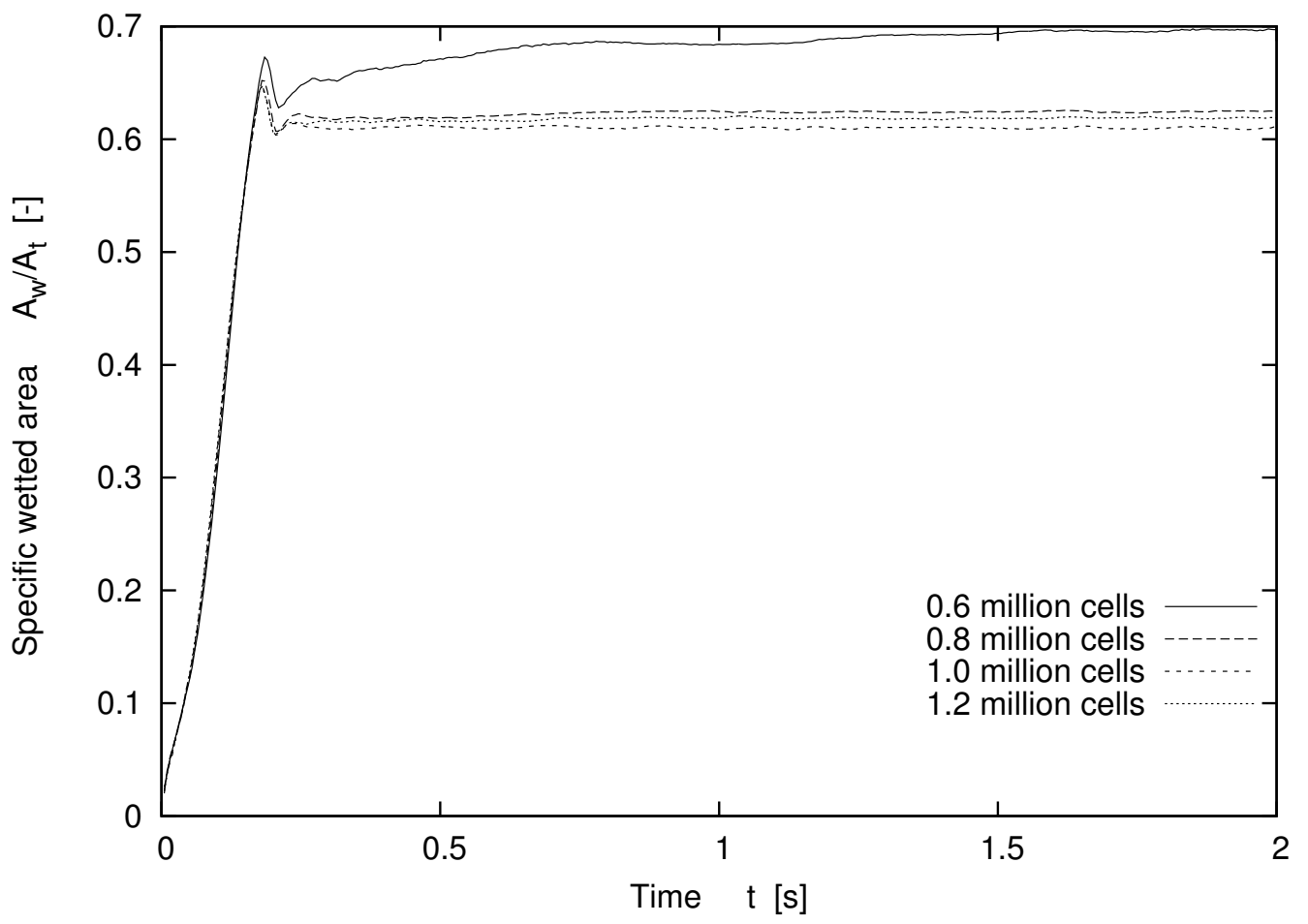

Figure A.15: Specific wetted area against time for static mesh $\theta=60^{\circ}, R e_{l}=156.85$

\section{Appendix A. Static Mesh Independence Checks}

Mesh independence checks were carried out on the highly refined static grid to ensure that the results were not affected by the choice of mesh. It was observed that the wetted area of the plate was highly dependent upon the flow parameters and therefore, this variable was used as a good indication to mesh dependency. Simulations were run initially with a mesh of 0.6 million cells, increasing the number of cells in subsequent calculations until minimal difference in the solution was observed. Figure A.15 plots the specific wetted area against time for $\theta=60^{\circ}$ and $R e_{l}=156.85$. It can be seen that there was very little difference in the solutions when using meshes of $0.8,1.0$ and 1.2 million cells. It was established that a cell count of 1.0 million cells allowed mesh dependency errors to be minimised, whilst keeping run-times to a reasonable level. 


\section{Acknowledgment}

The authors acknowledge the use of the IRIDIS High Performance Com-

puting Facility, and associated support services at the University of Southampton, in the completion of this work.

Alix, P., \& Raynal, L. (2008). Liquid distribution and liquid hold-up in modern high capacity packings. Chemical Engineering Research and Design, $86,585-591$.

Berberović, E., van Hinsberg, N. P., Jakirlić, S., Roisman, I. V., \& Tropea, C. (2009). Drop impact onto a liquid layer of finite thickness: Dynamics of the cavity evolution. Physical Review E, 79, 036306.

Brackbill, J., Kothe, D., \& Zemach, C. (1992). A continuum method for modeling surface tension. Journal of Computational Physics, 100, 335354.

Brambilla, P., \& Guardone, A. (2013). Automatic tracking of corona propagation in three-dimensional simulations of non-normal drop impact on a liquid film. Computing, (pp. 1-10).

Chasanis, P., Lautenschleger, A., \& Kenig, E. Y. (2010). Numerical investigation of carbon dioxide absorption in a falling-film micro-contactor. Chemical Engineering Science, 65, 1125-1133.

Cooke, J., Gu, S., Armstrong, L., \& Luo, K. (2012). Gas-Liquid Flow on Smooth and Textured Inclined Planes. World Academy of Science, Engineering and Technology, 68, 1712-1719.

C.W. Hirt and B.D. Nichols (1981). Volume of fluid (VOF) method for the dynamics of free boundaries. Journal of Computational Physics, 39, 201 -225 .

Freguia, S., \& Rochelle, G. T. (2003). Modeling of CO2 capture by aqueous monoethanolamine. AIChE Journal, 49, 1676-1686.

Gu, F., Liu, C. J., Yuan, X. G., \& Yu, G. C. (2004). CFD simulation of liquid film flow on inclined plates. Chemical Engineering 83 Technology, 27, 1099-1104. 
Haroun, Y., Legendre, D., \& Raynal, L. (2010). Direct numerical simulation of reactive absorption in gas-liquid flow on structured packing using interface capturing method. Chemical Engineering Science, 65, 351-356.

Ho, C. D., Chang, H., Chen, H. J., Chang, C. L., Li, H. H., \& Chang, Y. Y. (2011). CFD simulation of the two-phase flow for a falling film microreactor. International Journal of Heat and Mass Transfer, 54, 37403748 .

Hoffmann, A., Ausner, I., Repke, J. U., \& Wozny, G. (2005). Fluid dynamics in multiphase distillation processes in packed towers. Computers $\&$ Chemical Engineering, 29, 1433-1437.

Hoffmann, A., Ausner, I., Repke, J. U., \& Wozny, G. (2006). Detailed investigation of multiphase (gas-liquid and gas-liquid-liquid) flow behaviour on inclined plates. Chemical Engineering Research and Design, 84, 147-154.

Iso, Y., \& Chen, X. (2011). Flow Transition Behavior of the Wetting Flow Between the Film Flow and Rivulet Flow on an Inclined Wall. Journal of Fluids Engineering, 133, 091101.

Jasak, H. (1996). Error analysis and estimation for the finite volume method with applications to fluid flows.. Ph.D. thesis Imperial College London (University of London).

Jasak, H., \& Gosman, A. (2000). Automatic resolution control for the finitevolume method, Part 2: Adaptive mesh refinement and coarsening. $\mathrm{Nu}$ merical Heat Transfer: Part B: Fundamentals, 38, 257-271.

Kohrt, M., Ausner, I., Wozny, G., \& Repke, J. U. (2011). Texture influence on liquid-side mass transfer. Chemical Engineering Research and Design, 89, 1405-1413.

Lan, H., Wegener, J., Armaly, B., \& Drallmeier, J. (2010). Developing laminar gravity-driven thin liquid film flow down an inclined plane. Journal of Fluids Engineering, 132, 081301.

Nikolopoulos, N., Theodorakakos, A., \& Bergeles, G. (2007). Threedimensional numerical investigation of a droplet impinging normally onto a wall film. Journal of Computational Physics, 225, 322-341. 
Nusselt, W. (1916). Die Oberflchenkondensation des Wasserdampfes. Zeitschrift des Vereines Deutscher Ingenieure, 60, 541-546 and 569-575.

OpenCFD. (2011). OpenFOAM: The Open Source CFD Toolbox. Programmer's Guide Version 2.0.1. OpenCFD Ltd., Reading UK.

OpenCFD (2011). OpenFOAM: The Open Source CFD Toolbox. User Guide Version 2.0.1. OpenCFD Ltd., Reading UK.

Raynal, L., Ballaguet, J. P., \& Barrere-Tricca, C. (2004). Determination of mass transfer characteristics of co-current two-phase flow within structured packing. Chemical Engineering Science, 59, 5395-5402.

Raynal, L., Boyer, C., \& Ballaguet, J. (2004). Liquid holdup and pressure drop determination in structured packing with CFD simulations. The Canadian Journal of Chemical Engineering, 82, 871-879.

Raynal, L., \& Royon-Lebeaud, A. (2007). A multi-scale approach for CFD calculations of gas-liquid flow within large size column equipped with structured packing. Chemical Engineering Science, 62, 7196-7204.

Rusche, H. (2002). Computational Fluid Dynamics of Dispersed Two-Phase Flows at High Phase Fractions. Ph.D. thesis Imperial College, University of London.

Szulczewska, B., Zbicinski, I., \& Górak, A. (2003). Liquid flow on structured packing: CFD simulation and experimental study. Chemical Engineering \& Technology, 26, 580-584.

Theodorakakos, A., \& Bergeles, G. (2004). Simulation of sharp gas-liquid interface using VOF method and adaptive grid local refinement around the interface. International Journal for Numerical Methods in Fluids, 45, 421-439.

Valluri, P., Matar, O. K., Hewitt, G. F., \& Mendes, M. A. (2005). Thin film flow over structured packings at moderate Reynolds numbers. Chemical Engineering Science, 60, 1965-1975.

Xu, Y. Y., Paschke, S., Repke, J. U., Yuan, J. Q., \& Wozny, G. (2008). Portraying the Countercurrent Flow on Packings by Three-Dimensional Computational Fluid Dynamics Simulations. Chemical Engineering 8 Technology, 31, 1445-1452. 